\title{
Novel Non-orthogonal Multi-access Method for Multi-user MIMO with Antenna Number Modulation
}

\author{
SEYIT KARATEPE ${ }^{1}$, MUHAMMET KIRIK ${ }^{1}$, JEHAD M. HAMAMREH ${ }^{1}$ \\ ${ }^{1}$ S. Karatepe, M. Kirik, and J. M. Hamamreh are with WISLAB-TELENG for Wireless Research at the Department of Electrical-Electronics Engineering, \\ Antalya Bilim University, Antalya, Turkey. (web: https://sites.google.com/view/wislab // email: muhammet.kirik@ std.antalya.edu.tr). \\ Corresponding author: Muhammet Kirik (e-mail: muhammet.kirik@std.antalya.edu.tr).
}

The Matlab simulation codes used to generate the results in this paper can be found at https://researcherstore.com with the name MU-MIMO-ANM-AAS. This research was partly funded by TUBITAK under Grant/Award Number $119 E 392$.

\begin{abstract}
Multiple Input Multiple Output with Antenna Number Modulation and Adaptive Antenna Selection (MIMO-ANM-AAS) has recently been proposed as an effective transmission method that can make the selection of antennas to be both data and channel dependent simultaneously, which can result in having a much more improved performance in terms of reliability compared to index/spatial modulation-based schemes. However, one of the main undesirable features of MIMO-ANM-AAS, which is also the case with spatial modulation, is the fact that not all the available antennas are utilized for data transmission as some of these antennas are made inactive. To overcome this problem and utilize all available antennas, MultiUser MIMO-ANM-AAS is proposed in this paper as a novel non-orthogonal multi-access data transmission method that targets serving multiple users by dedicating the antennas used for implementing ANM to serve a far user, whereas the remaining antennas are used to send data for a near user. The fundamental idea behind the proposed scheme is to serve a far user, who is located in a distant location from the transmitter, by the antennas that are defined and utilized by the ANM modulation, while the remaining antennas are deployed to transmit a lower power data stream that is intended to be sent to a near user, who is located in a close proximity from the transmitter. By implementing this method, the near user can simply be served by allocating low transmission power to the antennas that are not used by ANM, whereas the far user is served by the antennas involved in the ANM modulation. By using such a transmission scheme, two users can be served simultaneously by a single MIMO-ANM architecture, and the obtained system reliability of the transmitted data stream for far user can be enhanced, while the capabilities of the antennas not used by ANM are not wasted, but instead utilized to serve an additional user, thus resulting in improving the overall spectral efficiency of the system. In this paper, the concepts of MU-MIMO-ANM-AAS are established on the basis of conventional MIMO using binary phase shift keying (BPSK) symbol modulation over a Rayleigh fading channel. The validity of the system is proven by exhibiting both theoretical analysis and computer simulations' results. Considering the acquired results, it can be said that the proposed MU-MIMO-ANMAAS is an effective scheme that can satisfy the requirements of multi-user cases for future wireless systems demanding higher reliability and, better spectral efficiency.
\end{abstract}

INDEX TERMS MIMO-ANM, MIMO-ANM-AAS, MU-MIMO-ANM-AAS, Nonorthogonal, antenna number modulation, multi-user, wireless communication, $6 \mathrm{G}$, future wireless networks

\section{INTRODUCTION}

W ITHIN the growing demand on the highly used internet services, capacities of the new generation wireless systems are expected to be increased in terms of data rate, spectral efficiency and power efficiency [1]-[5]. In the passing years, it is theoretically and practically proven that the Multiple Input Multiple Output (MIMO) systems can significantly increase the capacity of wireless systems to 
satisfy the aforementioned requirements [6]-[8]. However, considering the exponential growth in the number of mobile devices, sensors, and IoT technologies, data traffic in the future does not seem to be satisfiable with the single user, point to point data transmission techniques such as MIMO [9].

In this manner, Multi-User Multiple Input Multiple Output (MU-MIMO) systems have drawn a lot of attention throughout the passing years [10]. The basic idea behind the MUMIMO systems is that creating a MIMO system with a channel matrix, where each user's data can only be precoded by that user's transmitter antennas [11]. In general, this separate precoding process between the transmitter antennas and users is achieved by the orthogonalizing the channel. In this way, communication between the transmit antennas and users can be operated in different time and frequency resources [12]. However, serving different users in different time-frequency resources is not a preferable option, as it may cause a potential decreasement in the system. On the other hand, even though serving several users in the same timefrequency resource leads to the system to achieve higher rates [13], [14], as a trade off, it causes Multi-User Interference (MUI) [15].

There has been many studies in the past to alleviate the effects of MUI in literature [16]-[20]. Within the developments in the multiple access channel technologies, the MUI is aimed to be decreased by utilizing multiple antenna receivers that exploit advanced multi-user detection (MUD) techniques for the uplink with an increased receiver diversity trade off [21], [22]. However, due to the increased receiver complexity that is emerged because of the MUD operations, new design challenges arise at downlink to eliminate this effect. Even though such technologies as dirty paper coding (DPC) technique is proposed in many studies in the literature [23]-[25] to minimize the effect of MUI, its capabilities are not satisfactory in practice because of its high computational complexity. Another approach to overcome the receiver complexity at the downlink without getting affected by the drawbacks of computational complexity is the precoding, which is operated by providing the channel state information of the receiver to transmitter [26], [27].

There is a recent study named as MIMO with Antenna Number Modulation (MIMO-ANM) [28] proposed in the literature for addressing the drawbacks of spatial modulation [29]-[31]. According to this scheme, the additional data bits are sent by exploiting the number of antennas instead of their indices, which is achieved by letting one portion of the data to determine the number of antennas to be used, while the other portion is transmitted by the corresponding antennas by using conventional PSK/QAM modulation. By creating such an environment, it is aimed to make the antenna selection process both channel and data dependent on the contrary of SM. However, one of the main undesirable features of this scheme, which is also the case with spatial modulation, is the fact that not all the available antennas are utilized for data transmission as some of these antennas are made inactive.
In this paper, a novel multi-user adaptive method called "Multi-User MIMO-ANM-AAS" is proposed by inheriting the capabilities of [28] to satisfy such cases that requires multiuser service without getting affected by the aforementioned problems. In this method, while the user, who is located in a far location from the transmitter (Frank) is served by exploiting the merits of conventional MIMO-ANM, which relies on the idea of activating the transmit antennas according to incoming data of far user for the transmission, another user, who is located in a near location (Natalie) is aimed to be served by those antennas that are not deployed for the transmission of far user. Such a transmission technique results in serving multiple users at the same time with low receiver complexity, high spectral efficiency, and low BER. Consequently, the proposed scheme is expected to be a good candidate for the future $5 \mathrm{G}$ and beyond wireless networks that require multi-user services.

The organization of this paper in the following sections are indicated as follows. System model of MU-MIMO-ANMAAS and its merits are explained in detail in section II. Performance analysis of the proposed scheme is presented in section III. Simulation results of the regarding scheme are exhibited in section IV with a commentating discussion. Lastly, in section V, paper is finalized with a conclusion.

\section{SYSTEM MODEL}

In this section, structure of the MU-MIMO-ANM-AAS is deeply explained, where a multi-user MIMO scheme with two receivers under Rayleigh fading channel is considered. Number of antennas at the transmission side is considered to be $T$, and at the reception sides numbers of antennas for the user who is near to the transmitter and far to the transmitter are considered as $R_{n}$ and $R_{f}$, respectively. In this study, existence of an additional receiver, which is located in a nearer location to the transmitter for another user, is considered to exhibit the multi-user adaptivity of the proposed MU-MIMOANM-AAS. For simplicity, number of receiving antennas for both near user and far user are considered to be singular, i.e., $R_{n}=1, R_{f}=1$. $^{1}$

The focus of proposed data transmission scheme MUMIMO-ANM-AAS is to serve more than one user by exploiting the merits of ANM [28]. While the data that has to be transmitted to the far receiver is transmitted by the antennas that exploit the features of ANM in high power, remaining transmit antennas that do not exploit the features of ANM can serve another receiver that is nearer to the transmitter in low power. With this scheme, ANM can be deployed in such cases that require high spectral efficiency and low bit error rate for multi-user serving transmission environments.

\footnotetext{
${ }^{1}$ Notations: We denote vectors by bold-small letters, matrices by boldcapital letters, scalar numbers by non-bold capital letters, and complex numbers or elements indexing by non-bold small letters based on the context. Also all the logarithmic functions are based 2 unless stated otherwise.
} 


\section{A. DESIGN OF THE TRANSMISSION END OF MU-MIMO-ANM-AAS}

The general structure of the proposed MU-MIMO-ANMAAS transmitter is shown in Fig. 1.

Procedure starts with dividing the incoming bit stream, $N=N_{1}+N_{2}$, into two sub-streams. Portion of the bit stream that is located at the beginning of the data stream is the part that is responsible of the mapping of signal constellation points, $\left(N_{1}\right)$, which is determined by the signal constellation modulation order $N_{1}=\log (M)$. In this regard, this portion is named as "main bits". Portion of the bit stream that is located at the end of the data stream is the part that is responsible of the mapping of antenna activation patterns, $\left(N_{2}\right)$, which is determined by number of available transmit antennas $T$ as per this formula $N_{2}=\log (T)$. In this regard, this portion is named as "ANM Bits".

In the next step each symbol in the main bits sub-stream is modulated by conventional BPSK and each symbol in ANM bits sub-stream is exploited in the decision process to define the number of active antennas that will be used for the transmission of main bits. This decision is made by using a look-up table that maps ANM bits sub-stream for specific bit combinations. The illustration of the mapping process for such a case where the total number of antennas for the transmission is four $(T=4)$ is given in Table 1 .

TABLE 1. MU-MIMO-ANM-AAS mapper with $N_{2}=2$ bits \& $T=4$ antennas, where the final active antenna pattern is determined by the largest channel gains among all possible antenna numbers

\begin{tabular}{|c|c|}
\hline ANM bits $\left(N_{2}\right)$ & Possible Active Antennas Pattern (v) \\
\hline$\left[\begin{array}{ll}0 & 0\end{array}\right]$ & {$\left[\begin{array}{llll}1 & 0 & 0 & 0\end{array}\right]$} \\
\hline $\left.\begin{array}{lll}0 & 0\end{array}\right]$ & $\left.\begin{array}{llll}0 & 1 & 0 & 0\end{array}\right]$ \\
\hline$[00]$ & {$\left[\begin{array}{llll}0 & 0 & 1 & 0\end{array}\right]$} \\
\hline$\left[\begin{array}{ll}0 & 0\end{array}\right]$ & {$\left[\begin{array}{llll}0 & 0 & 0 & 1\end{array}\right]$} \\
\hline$\left[\begin{array}{ll}0 & 1\end{array}\right]$ & {$\left[\begin{array}{llll}1 & 1 & 0 & 0\end{array}\right]$} \\
\hline$\left[\begin{array}{ll}0 & 1\end{array}\right]$ & {$\left[\begin{array}{llll}1 & 0 & 1 & 0\end{array}\right]$} \\
\hline$\left[\begin{array}{ll}0 & 1\end{array}\right]$ & {$\left[\begin{array}{llll}1 & 0 & 0 & 1\end{array}\right]$} \\
\hline$\left[\begin{array}{ll}0 & 1\end{array}\right]$ & {$\left[\begin{array}{llll}0 & 0 & 1 & 1\end{array}\right]$} \\
\hline$\left[\begin{array}{ll}0 & 1\end{array}\right]$ & {$\left[\begin{array}{llll}0 & 1 & 0 & 1\end{array}\right]$} \\
\hline$\left[\begin{array}{ll}0 & 1\end{array}\right]$ & {$\left[\begin{array}{llll}0 & 1 & 1 & 0\end{array}\right]$} \\
\hline [1 0 ] & {$\left[\begin{array}{llll}1 & 1 & 1 & 0\end{array}\right]$} \\
\hline [1 0 0] & {$\left[\begin{array}{llll}1 & 1 & 0 & 1\end{array}\right]$} \\
\hline [1 0 l & {$\left[\begin{array}{llll}1 & 0 & 1 & 1\end{array}\right]$} \\
\hline$\left[\begin{array}{lll}1 & 0\end{array}\right]$ & {$\left[\begin{array}{llll}0 & 1 & 1 & 1\end{array}\right]$} \\
\hline$\left[\begin{array}{ll}1 & 1\end{array}\right]$ & {$\left[\begin{array}{llll}1 & 1 & 1 & 1\end{array}\right]$} \\
\hline
\end{tabular}

As it can be seen from Table 1, if the ANM bits symbol is "00", then the number of active antennas is one; if ANM bits symbol is "01", then the number of active antennas is two; if ANM bits symbol is "10", then the number of active antennas is three; and if ANM bits symbol is "11", then the number of active antennas is four. Determination of the mapping numbers of each antenna is operated by designing a system that includes $T=2^{N_{2}}$ transmit antennas, where $\mathrm{N}_{2}$ is the each symbol in ANM bits sub-stream. For a fair comparison between MIMO-ANM-AAS and MU-MIMO-
ANM-AAS, and to observe the multi-user capabilities of the proposed scheme, a setup that consists of four transmit antennas $(T=4)$, one receive antenna for far user $\left(R_{f}=1\right)$, and one receive antenna for near user $\left(R_{n}=1\right)$ is considered in the description of the proposed scheme throughout the coming sections of this paper.

In the following step, channel capacities of each antenna is determined to maintain the adaptive antenna selection nature of ANM in the multi-user case. In this regard, the channel capacities of transmit antennas are sorted in a descending order from the highest channel capacity offering antennas to lowest channel capacity offering antennas. In this way, the proposed scheme does not become only data dependent such as MIMO-SM but it also becomes channel dependent. Possible antenna activation patterns for this adaptive antenna selection procedure is given in Table 1 .

After the channel capacities of the transmit antennas are defined and sorted from high quality to low quality, the procedure proceeds as follows. Symbols in the main bits substream, which are modulated by BPSK for the data transfer of the far user are transmitted by exploiting the high channel quality offering antennas with high power. The mathematical expression for the transmission of main bits for the receiver that is located in a far point from the transmitter is given as follows:

$$
y_{f}=\sqrt{\frac{P_{h i g h}}{V}} \times \mathbf{h}_{\mathbf{h q}} \times \mathbf{v} \times\left(x_{f}\right)+w
$$

In this formula, $P$ is the transmit power per data symbol. $V=\|\mathbf{v}\|_{2}=\sum_{i=1}^{T} v_{i}^{2}$ is the total number of active antennas selected for the transmission of the far user's data out of $T$ available antennas in each channel use. $\mathbf{h}=$ $\left[h_{11}, h_{12}, h_{13}, h_{14}\right] \in \mathbb{C}^{1 \times 4}$ is the flat fading channel vector in which each element represents a circularly symmetric complex Gaussian channel coefficient with zero mean and unity variance, corresponding to the response between $R^{t h}$ receive antenna and $T^{t h}$ transmit antenna. The activation pattern vector for the antennas that are deployed to transmit the far user's data is defined as $\mathbf{v} \in \mathbb{R}^{4 \times 1}$. Elements of this vector may vary as zeros for inactive antennas and ones for active antennas according to the condition of each corresponding antenna, which are determined according to $N_{2}$ bits and the mapping/lookup process given in Table 1 . $x_{f}$ is the each symbol of main bits sub-stream, which is modulated by BPSK, and $w$ is the white Gaussian noise, which also includes the low powered signal that comes from the low quality offering antennas for near user.

After the transmission of the far user's data, main bits, is completed by deploying the high channel quality offering antennas, the remaining low channel quality offering antennas are deployed to transmit another bit stream, which is modulated by BPSK and intended to be sent a nearer user. The mathematical expression of the transmission of this secondary data stream for the receiver that is located in a near 


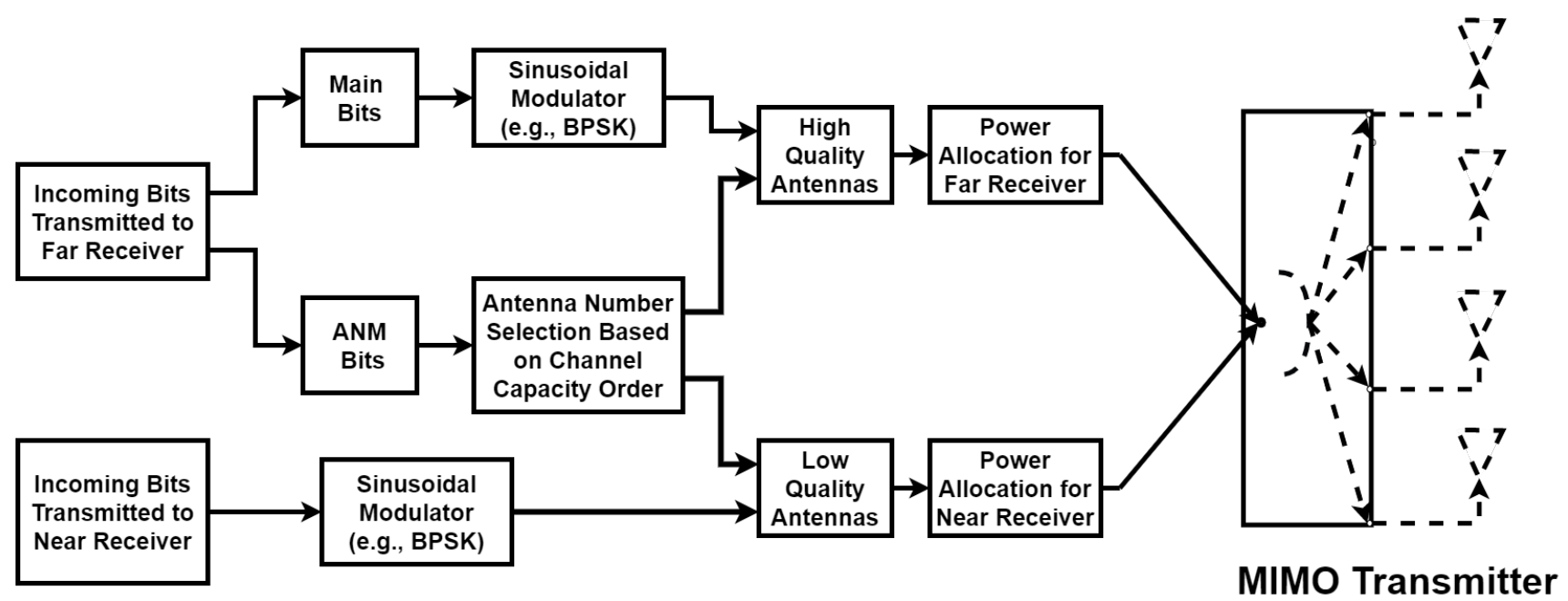

FIGURE 1. Transmitter Structure of MU-MIMO-ANM-AAS

point from the transmitter is given as follows:

$$
y=y_{f}+y_{n},
$$

$$
\begin{aligned}
y=\sqrt{\frac{P_{\text {high }}}{V}} \times \mathbf{h}_{\mathbf{h q}} \times \mathbf{v} \times\left(x_{f}\right)+ \\
\sqrt{\frac{P_{l o w}}{Z}} \times \mathbf{h}_{\mathbf{l q}} \times \zeta \times\left(x_{n}\right)+w .
\end{aligned}
$$

The received data by the near user's receiver, $y$, is the summation of the data that comes from both the high channel quality offering antennas as stated in (2) and (3), which is the symbols of main bits and intended to be sent to the far user; and the low channel quality offering antennas, which is the symbols of secondary data stream that is actually intended to be sent to the near user as the near user's receiver is in the power range of both the high powered signal and the low powered signal.

$$
y_{n}=y-y_{f}
$$

$$
\begin{aligned}
& y_{n}=\sqrt{\frac{P_{\text {high }}}{V}} \times \mathbf{h}_{\mathbf{h q}} \times \mathbf{v} \times\left(x_{f}\right)+ \\
& \sqrt{\frac{P_{\text {low }}}{Z}} \times \mathbf{h}_{\mathbf{l q}} \times \zeta \times\left(x_{n}\right)-\left[\sqrt{\frac{P_{h i g h}}{V}} \times \mathbf{h}_{\mathbf{h q}} \times \mathbf{v} \times\left(x_{f}\right)\right]+w
\end{aligned}
$$

To distinguish the near user's low powered signal that is sent by the low channel quality offering antennas, the successive interference cancellation (SIC) algorithm is used as stated in (4) and (5). The final mathematical expression of the transmission of the secondary data stream for the receiver that is located in a near point from the transmitter is given in (6).

$$
y_{n}=\sqrt{\frac{P_{\text {low }}}{Z}} \times \mathbf{h}_{\mathbf{l q}} \times \zeta \times\left(x_{n}\right)+w .
$$

In this formula, $Z=\|\zeta\|_{2}=\sum_{i=1}^{T} \zeta_{i}^{2}$ is the total number of active antennas selected for the transmission of the near user's data out of $T$ available antennas in each channel use. The activation pattern vector for the antennas that are deployed to transmit the near user's data is defined as $\zeta \in \mathbb{R}^{4 \times 1} . x_{n}$ is the each symbol of secondary data stream, which is modulated by BPSK.

Example: Consider a group of people consisting of three friends; Ted, Frank, and Natalie. Ted wants to transmit two different data streams to Frank and Natalie with four transmit antennas $(T=4)$, while Frank and Natalie have only one receive antenna $\left(R_{f}=1, R_{n}=1\right)$. In this group of people, location of Natalie is nearer to location of Ted, while Frank is located in a longer distance. The regarding data streams to be sent for both far receiver and near receiver is given in Fig. 2.

Incoming Data Stream for Far Receiver

\begin{tabular}{|l|l|l|l|l|l|l|l|l|l|l|l|l|}
\hline $\mathbf{1}$ & $\mathbf{0}$ & $\mathbf{0}$ & $\mathbf{1}$ & $\mathbf{1}$ & $\mathbf{0}$ & $\mathbf{0}$ & $\mathbf{1}$ & $\mathbf{0}$ & $\mathbf{0}$ & $\mathbf{1}$ & $\mathbf{1}$ \\
\hline \\
\hline
\end{tabular}

Incoming Data Stream for Near Receiver

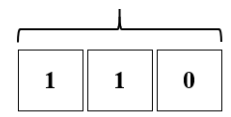

FIGURE 2. Incoming data streams to be transmitted to the far receiver, Frank, with main bits and ANM bits separation and near receiver, Natalie, under BPSK modulation.

During the transmission process, channel quality ranking of each antenna between Ted and Frank is assumed to be $\left|h_{1}\right|>\left|h_{4}\right|>\left|h_{2}\right|>\left|h_{3}\right|$. This means that the antenna activation pattern of the transmission of Frank's data will be in a descending order as $T_{1}>T_{4}>T_{2}>T_{3}$. After the 
antenna activation order is determined, each symbol in the main bits sub-stream is transmitted to the far receiver by a specific number of antennas that their number is defined by the ANM bits sub-stream and offer the best possible channel quality. After this step, remaining antennas in the system that was not deployed for the transmission of the far user's data, are deployed for transmitting the secondary data stream that serves to near user. In that manner, the step by step explanation of Fig. 2 is given as follows.

After the main bits, $N_{1}=\log (M)$, and ANM bits, $N_{2}=\log (T)$, separation is achieved in the incoming data stream for the far receiver, the data transmission from Ted to Frank starts with the first symbol of main bits sub-stream 1. As the first symbol in the ANM bits sub-stream is 10 , the transmission of 1 must be operated by the three transmit antennas that offer the highest possible channel quality, which are $T_{1}, T_{4}, T_{2}$. After the transmission of the first symbol of main bits sub-stream is achieved by these three antennas, the remaining antenna, (ie., $T_{3}$ ) is deployed to convey the first symbol of the secondary incoming data stream 1 that is sent to near receiver Natalie. This process is illustrated in Fig. 3-a.

The second symbol of the main bits group is 0 , and it has to be sent to far user by deploying two antennas as the second symbol of the ANM bits sub-stream is 01. In this case, since the two highest channel quality offering antennas are $T_{1}$ and $T_{4}$, these two antennas are deployed to serve the far user Frank, while the remaining antennas $T_{2}$ and $T_{3}$, which their channel qualities are relatively lower than $T_{1}$ and $T_{4}$, are deployed to convey the second symbol of the secondary incoming data stream 1 that is sent to near receiver Natalie. Illustration of this procedure is provided in Fig. 3-b.

Number of active antennas that is deployed to transmit the third symbol of the main bits sub-stream 0 , is determined by the third symbol of ANM bits sub-stream which is 00. This means the activation of one antenna that can offer the highest possible channel quality. In this manner, $T_{1}$ is deployed to serve the far user Frank, while the remaining three antennas $T_{4}, T_{2}$, and $T_{3}$ are deployed to serve the near user Natalie to transmit 0 , which is the third symbol of the secondary data stream. Fig. 3-c shows the visual illustration of this operation.

The last symbol of the main bits sub-stream is 1 and it has to be transmitted by all the four antennas in the transmission side, as the last symbol of the ANM bits sub-stream is 11 . Since the priority of the transmitter is to convey the data that has to be transmitted to far receiver, the near receiver is not served in such a case, where all the antennas are deployed for the transmission of symbol of main bits sub-stream. This is visually shown in Fig. 3-d.

\section{B. DESIGN OF THE RECEPTION END OF MU-MIMO-ANM-AAS FROM FRANK'S PERSPECTIVE}

The receiver structure of the proposed MU-MIMO-ANMAAS is given in Fig. 4 from Frank's perspective.

For the decoding of Frank's data, which consists of main bits and ANM bits, and individual (separate) detection is adopted to detect the data bits conveyed by both antenna number and conventional $M$-ary signal constellation symbols.

To decode ANM bits and main bits separately, a maximum likelihood (ML) detector is used for the estimation of the active antennas' pattern and signal constellation points. The ML detector for these two cases are given in the following:

$$
\begin{aligned}
& J_{v}=\min _{\hat{v}}\left(\left\|y_{f}-\left(\sum_{i=1}^{\hat{v}} h_{1 i}\right) x_{f}\right\|^{2}\right), \\
& J_{x f}=\min _{\hat{x_{f}}}\left(\left\|y_{f}-\left(\sum_{i=1}^{v} h_{1 i}\right) \hat{x_{f}}\right\|^{2}\right),
\end{aligned}
$$

where $v \in 1,2,3,4$ is the possible number of active transmit antennas, $y_{f i} \in y_{f 1}, y_{f 2}, y_{f 3}, y_{f 4}$ is the received signal according to the possible antenna number usage, and $\hat{x_{f i}}$ is the estimated BPSK symbol.

\section{DESIGN OF THE RECEPTION END OF MU-MIMO-ANM-AAS FROM NATALIE'S PERSPECTIVE}

The receiver structure of the proposed MU-MIMO-ANMAAS is given in Fig. 5 from Natalie's perspective.

For the decoding of Natalie's data an ML detector is used to estimate the transmitted bits at the receiver. However, since the near receiver is capable of receiving both the data that is meant to be sent to the far user and her, in this demodulation, an SIC algorithm is implemented to eliminate the confusion for Natalie. The ML detector for this case is given as follows:

$J_{x_{n}}=\min _{\hat{x_{f}}}\left(\| y-\left[\left(\sum_{i=1}^{v} h_{h q_{1 i}}\right) x_{f}+\left(\sum_{i=1}^{\zeta} h_{l q_{1 i}}\right) x_{n} \|^{2}\right]\right)$,

\section{PERFORMANCE ANALYSIS}

In this section, performance of the proposed MU-MIMOANM-AAS is analysed and acquired results are formulated in a mathematical form. In this manner, the statistics of the effective instantaneous SNR need to be calculated for both Frank and Natalie, given by $\gamma_{f}=\frac{\left\|H_{f i}\right\|^{2} \times P_{f}}{\sigma_{f}^{2}}$ and $\gamma_{n}=\frac{\left\|H_{n i}\right\|^{2} \times P_{n}}{\sigma^{2}}$, respectively. Also, the distribution functions of Frank and Natalie that are associated with their effective corresponding channels are presented in this section to determine the distribution of $\gamma_{f}$ and $\gamma_{n}$, and interrogate the multi-user performance of proposed scheme.

\section{A. STATISTICS OF FRANK'S EFFECTIVE SNR}

The nature of ANM leads the effective distribution to be changed with respect to ANM bits sub-stream, which is dependent on Frank's data. The reason behind this is that the antenna activation pattern of Frank to transmit each symbol of main bits sub-stream changes according to the ANM bits sub-stream and each antenna in this activation pattern are specifically selected to offer the highest possible channel capacity while the remaining antennas are excluded. In order to obtain the distributions of the amplitude and power of 
[a]

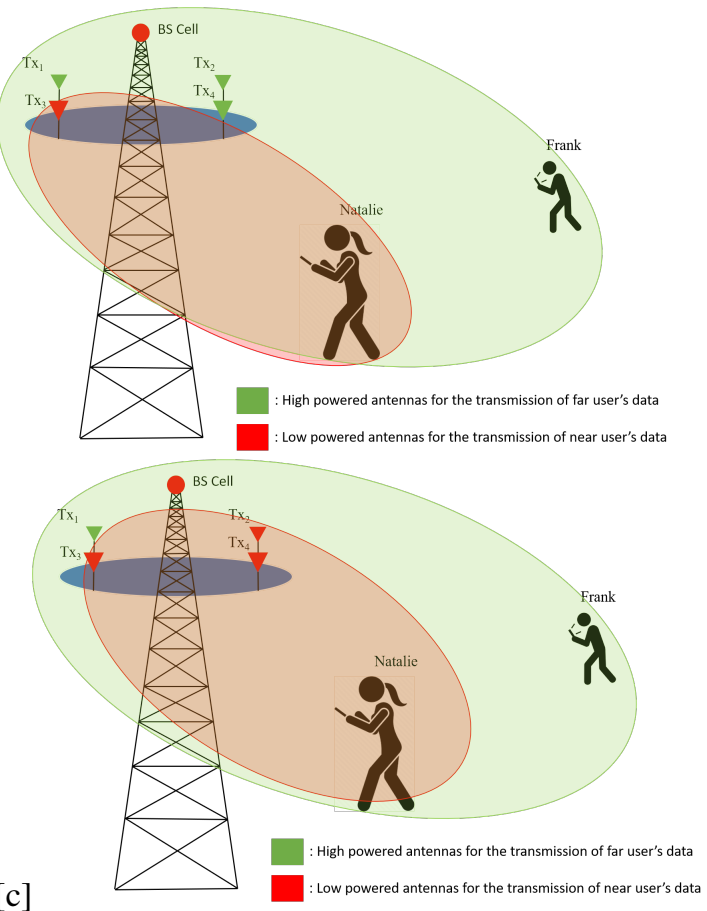

[b]

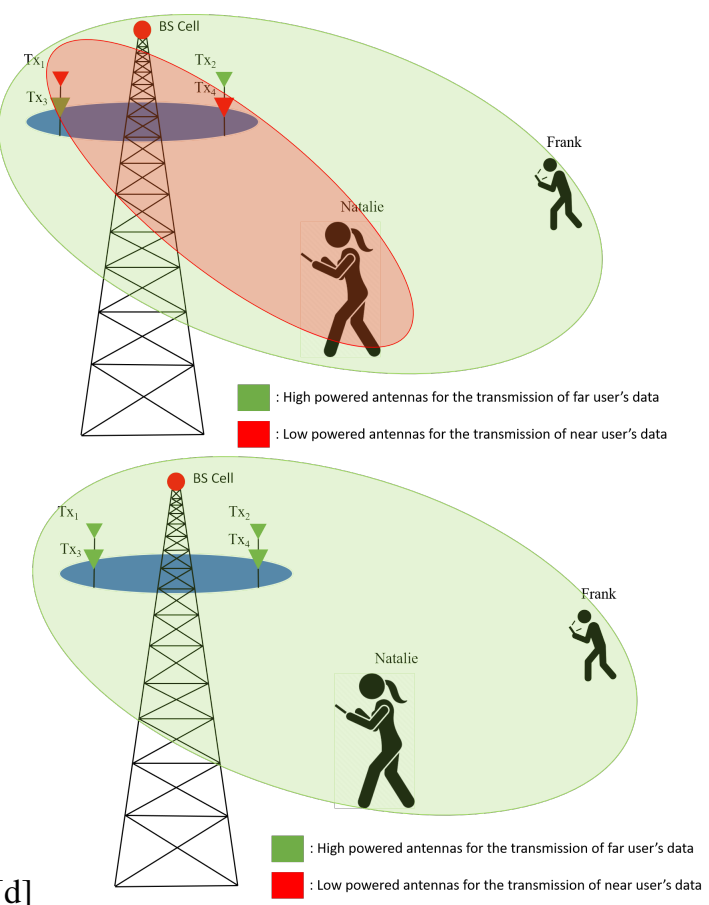

FIGURE 3. Simple Visualization of the Transmission of each Symbol for Different Antenna Activation Patterns

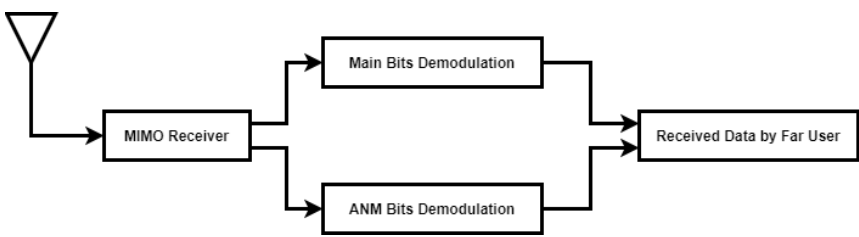

FIGURE 4. Receiver Structure of MU-MIMO-ANM-AAS for Far User Frank.

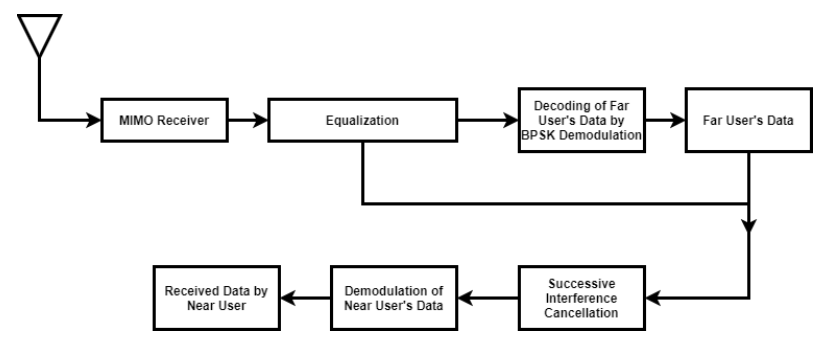

FIGURE 5. Receiver Structure of MU-MIMO-ANM-AAS for Near User Natalie.

the faded channels, we used numerical data fitting methods in this paper. The distributions are obtained by simulating 32000 realizations generated from a standard Rayleigh distribution, which is acquired by taking the amplitude of a circularly symmetric Gaussian distribution with zero mean and unity variance. After this point, the proposed MU-MIMOANM-AAS is applied to define the number of antennas that is deployed to transmit Frank's data, and defined by the incoming ANM bits. Lastly, the distribution parameters that lead the fading amplitude and power of effective channels to behave in their best conditions are defined by utilizing the fitting tools.

The fact that the selection of transmit antenna pattern is determined by the ANM bits, corresponds to a random channel set with respect to receiver. For this reason the anticipated distribution is expected to be the original fading distribution, which is Rayleigh but with different scale factors and means. Observations from the fitting results prove that the effective distribution over the channels exhibits the same characteristics as Rayleigh with different scale factor, $\beta=$ 0.71 and means as shown in Fig. 6. Moreover, the effective channel power distribution over each channel is found to be exponential with mean parameter $\psi \cong 1$ as shown in Fig. 7 . Mathematical expression for the PDF of the effective channel fading amplitude, i.e., $\alpha_{f}=\left\|H_{f i}\right\|$ is given by the following formula:

$$
P_{\alpha_{f}}\left(\alpha_{f}\right)=\frac{\alpha_{f}}{\beta^{2}} \exp \left(-\frac{\alpha_{f}^{2}}{2 \beta^{2}}\right)
$$

where $\beta$ is the scale parameter of the obtained Rayleigh channel distribution. Also, $\Omega_{f}$ is the mean square variable of $\alpha_{f}$, ie., $\Omega_{f}=E\left\{\alpha_{f}^{2}\right\}$, which is also equal to $\psi$ obtained by fitting methods.

The PDF of $\psi_{f}$ can be determined by using a change of variables in the expression for the fading distribution 


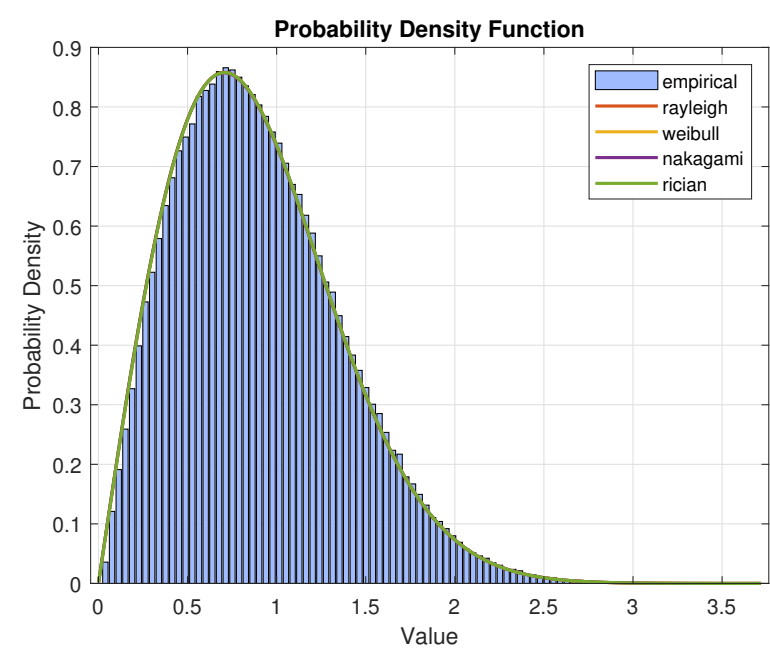

FIGURE 6. The amplitude distribution of the effective channels for Frank and Natalie using the proposed technique with scale parameter $\beta=0.71$

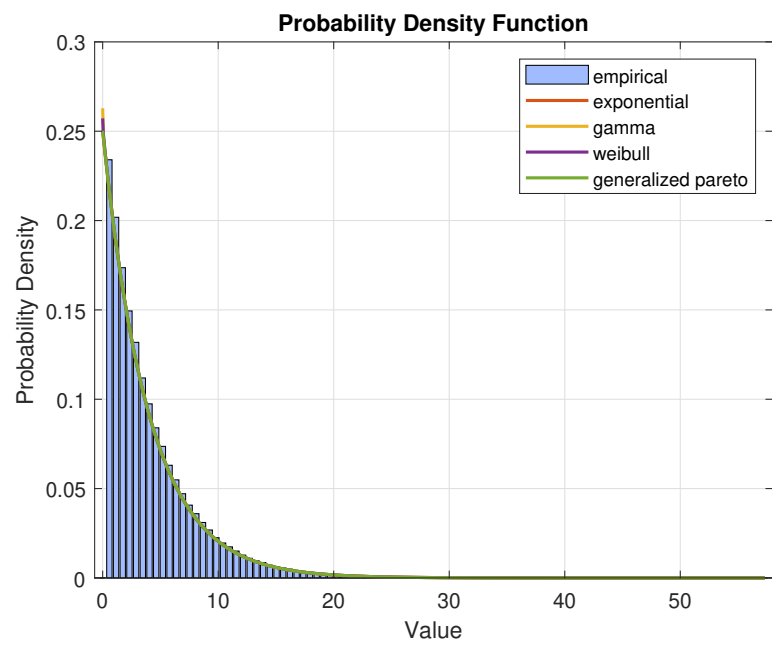

FIGURE 7. The power distribution of the effective channels for Frank and Natalie using the proposed technique with mean parameter $\psi \cong 1$

$P_{\alpha_{f}}\left(\alpha_{f}\right)$ of $\alpha_{f}$, yielding:

$$
P_{\gamma_{f}}\left(\gamma_{f}\right)=\frac{P_{\alpha}\left(\sqrt{\frac{\Omega_{f} \gamma_{f}}{\gamma_{f}}}\right)}{\frac{1}{\beta^{2}} \sqrt{\frac{\gamma_{f} \gamma_{f}}{\Omega_{f}}}}
$$

By considering the case of any antennas' activation pattern in MIMO-ANM scheme, the approximated PDF of the effective SNR, $P_{\gamma_{f}}\left(\gamma_{f}\right)$, can be given in an exponential distribution form. For the special case, when only one antenna is active, which corresponds to $\beta=0.71$, the instantaneous SNR distribution formula can be found as

$$
P_{\gamma_{f}}\left(\gamma_{f}\right)=\left(\frac{1}{\Omega_{f} \overline{\gamma_{f}}}\right) \exp \left(\frac{\gamma_{f}}{\Omega_{f} \overline{\gamma_{f}}}\right)
$$

\section{B. STATISTICS OF NATALIE'S EFFECTIVE SNR}

In order to define the distribution function of instantaneous SNR at Natalie under the effect of proposed technique, the fitting method is applied for the effective channel fading amplitude experienced by each transmit antenna similar to case of Frank's channel. Since the antenna activation process is operated by deploying the highest possible channel quality offering antennas for Frank, which corresponds to random antenna set for Natalie, the distribution is intuitively anticipated to be similar to the original assumed fading distribution i.e., Rayleigh. Acquired results prove the legitimacy of this intuitive anticipation and demonstrate the effective distribution of the channel amplitude is approximately Rayleigh distributed (same as the original one) with scale factor $\beta$ as shown in Fig. 6. Also, the effective channel power distribution over each channel is found to be exponential with mean parameter $\psi \cong 1$ as shown in Fig. 7. Mathematically, the amplitude channel distribution is derived by the following formula:

$$
P_{\alpha_{n}}\left(\alpha_{n}\right)=\frac{\alpha_{n}}{\beta^{2}} \exp \left(-\frac{\alpha_{n}^{2}}{2 \beta^{2}}\right)
$$

where $\Omega_{n}$ is the mean square variable of $\alpha_{n}$, ie., $\Omega_{n}=$ $E\left\{\alpha_{n}^{2}\right\}$, which is also equal to $\psi$ obtained by fitting methods.

The PDF of $\psi_{n}$ can be determined by using a change of variables in the expression for the fading distribution $P_{\alpha_{n}}\left(\alpha_{n}\right)$ of $\alpha_{n}$, yielding:

$$
P_{\gamma_{n}}\left(\gamma_{n}\right)=\frac{P_{\alpha}\left(\sqrt{\frac{\Omega_{n} \gamma_{n}}{\gamma_{n}}}\right)}{\frac{1}{\beta^{2}} \sqrt{\frac{\gamma_{n} \gamma_{n}}{\Omega_{n}}}}
$$

By considering the case of any antennas' activation pattern in MU-MIMO-ANM-AAS scheme, the approximated PDF of the effective SNR, $P_{\gamma_{n}}\left(\gamma_{n}\right)$, can be given in an exponential distribution form. For the special case, when three antenna is available for Natalie, which corresponds to $\beta=0.71$, the instantaneous SNR distribution formula can be found as

$$
P_{\gamma_{n}}\left(\gamma_{n}\right)=\left(\frac{1}{\Omega_{n} \overline{\gamma_{n}}}\right) \exp \left(\frac{\gamma_{n}}{\overline{\Omega_{n} \bar{\gamma}_{n}}}\right)
$$

Evaluations of the BER performances for both Frank and Natalie now can be achieved by exploiting the above calculated distribution functions.

\section{BER OF FRANK}

As it is proven in the previous sections, the PDF of the instantaneous SNR of Frank is Rayleigh. Under this condition, the BER performance of Frank for BPSK is given as follows:

$$
B E R_{f}=\frac{1}{2} \int_{0}^{\infty} \operatorname{erfc}\left(\sqrt{\gamma_{f}}\right) P_{\gamma_{f}}\left(\gamma_{f}\right) d_{\gamma_{f}}
$$


By substituting the PDF of the effective instantaneous SNR of Frank into (16), we get the following integration formula:

$$
B E R_{f}=\frac{1}{2} \int_{0}^{\infty} \operatorname{erfc}\left(\sqrt{\gamma_{f}}\right)\left(\frac{1}{\overline{\gamma_{f}}}\right) \exp \left(\frac{\gamma_{f}}{\overline{\gamma_{f}}}\right)
$$

The exact closed-form expression for Frank's BER can be expressed by solving the above integral as follows [28]:

$$
B E R_{f}=\frac{1}{2}\left(1-\frac{\overline{\gamma_{f}}}{\left(1+\overline{\gamma_{f}}\right)}\right)
$$

When the MU-MIMO-ANM-AAS is considered from Frank's perspective, there are two possible reasons for error. The first type of error is the one that occurs because of the detection of main bits, and the second one is that occurs during the detection of ANM bits.

The theoretical BER results that occur during the detection of ANM bits can be calculated by exploiting the (18). These calculations are derived an given as follows:

$$
B E R_{A N M-i}=\frac{T-1}{2 \times T} \times\left(1-\sqrt{\frac{\frac{\frac{3}{15} \times T_{a c t} \times E_{b}}{2 \times \beta^{2} \times N_{0}}}{\frac{\frac{3}{15} \times T_{a c t} \times E_{b}}{2 \times \beta^{2} \times N_{0}}+1}}\right)
$$

where $T$ is the number of transmit antennas in the system, $i$ is the iterator from 1 to $T, \beta$ is the scale factor for the antenna activation pattern, and $T_{a c t}$ is the number of active transmit antennas.

After the BER due to each case is calculated separately, the average value of these results is taken as the BER of the ANM Bits as given below:

$$
B E R_{A N M}=\frac{\sum_{i=1}^{4} B E R_{A N M-i}}{4} .
$$

The theoretical BER formulas of main bits for different number of active transmit antennas can be obtained from (20). The BER formulas of main bits are calculated and given as follows:

$$
B E R_{\text {Main }-i}=\frac{1}{2} \times\left(1-\sqrt{\frac{\frac{T_{a c t} \times E_{b}}{2 \times \beta^{2} \times N_{0}}}{\frac{T_{a c t} \times E_{b}}{2 \times \beta^{2} \times N_{0}}+1}}\right)
$$

After the BER results are calculated for each antenna activation case, the average value of the acquired results is taken as the BER of the main bits as given below:

$$
B E R_{\text {Main }}=\frac{\sum_{i=1}^{4} B E R_{\text {Main-i }}}{4} .
$$

At the last step, the average value of ANM bits substream's and main bits sub-stream's BER is calculated to find the theoretical total average BER of the MU-MIMO-ANMAAS for Frank as follows:

$$
B E R_{f}=\frac{2}{3} \times B E R_{A N M}+\frac{1}{3} \times B E R_{M a i n} .
$$

\section{BER OF NATALIE}

In the previous sections, it is proven that the PDF of the instantaneous SNR of Natalie exhibits the same characteristics as Frank, and remains Rayleigh. With this knowledge, it can be inferred that the BER performance of Natalie under the effect of proposed scheme is the same as conventional MIMO.

For BPSK modulation system, the BER of Natalie can be given as

$$
B E R_{n}=\frac{1}{2} \int_{0}^{\infty} \operatorname{erfc}\left(\sqrt{\gamma_{n}}\right) P_{\gamma_{n}}\left(\gamma_{n}\right) d_{\gamma n}
$$

By substituting the PDF of the effective instantaneous SNR of Natalie into (24), we get the following integration formula:

$$
B E R_{n}=\frac{1}{2} \int_{0}^{\infty} \operatorname{erfc}\left(\sqrt{\gamma_{n}}\right)\left(\frac{1}{\overline{\gamma_{n}}}\right) \exp \left(\frac{\gamma_{n}}{\overline{\gamma_{n}}}\right)
$$

The above integral can readily be solved, and its final solution yields an exact closed-form expression for Natalie's BER, which can be given as

$$
B E R_{n}=\frac{1}{2}\left(1-\frac{\overline{\gamma_{n}}}{\left(1+\overline{\gamma_{n}}\right)}\right)
$$

The theoretical BER formulas of Natalie's data stream for different number of transmit antennas can be obtained from (26). The BER formulas of this data stream are calculated and given as follows:

$B E R_{n-i}=\frac{T_{\text {inact }}+1}{T_{\text {inact }}} \times\left(1-\sqrt{\frac{\frac{E_{b}}{2 \times T_{\text {inact }}^{2} \times \beta^{2} \times N_{0}}}{\frac{E_{b}}{2 \times T_{\text {inact }}^{2} \times \beta^{2} \times N_{0}}+1}}\right)$

where $T_{\text {inact }}$ is the number of transmit antennas that are not deployed in the transmission of Frank's data.

After the BER results are calculated for each antenna activation case, the average value of the acquired results is taken as the BER of the Natalie as given below:

$$
B E R_{n}=\frac{\sum_{i=1}^{4} B E R_{n-i}}{4} .
$$

\section{SIMULATION RESULTS}

In this section, simulation results of the proposed MUMIMO-ANM-AAS scheme are exhibited in terms of BER and spectral efficiency by conducting Monte-Carlo simulations over a wireless Rayleigh fading channel to demonstrate and validate the effectiveness of proposed scheme.

The reason why the simulations of the proposed scheme are considered in these parameters is focusing on the merits of the system without loss of generality, and creating an atmosphere that makes the comparison of the MU-MIMOANM-AAS easier with other competitive schemes in the literature. The simulation parameters for the proposed scheme is given in Table 2 . 
TABLE 2. Simulation Parameters

\begin{tabular}{|c|c|}
\hline Modulation Type & BPSK $(M=2)$ \\
\hline Number of Symbols & $10^{6}$ per iteration \\
\hline Number of Transmit Antennas & 4 \\
\hline Number of Receive Antennas for Far User & 1 \\
\hline Number of Receive Antennas for Near User & 1 \\
\hline Number of available antennas for ANM & 2 \\
\hline Number of ANM mapping bits & Block Rayleigh fading \\
\hline Wireless channel &
\end{tabular}

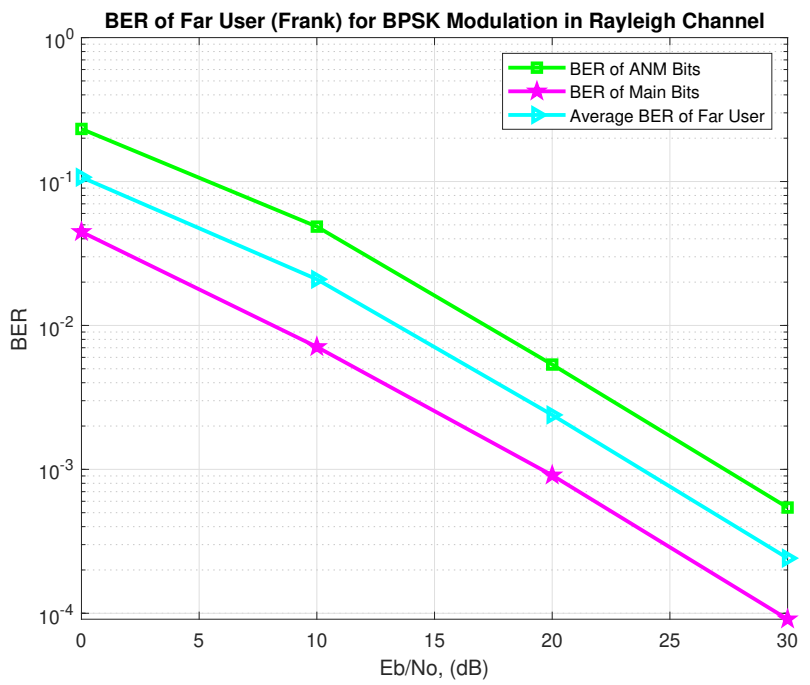

FIGURE 8. Simulated BER Performance of the Proposed MU-MIMO-ANM-AAS scheme Under BPSK Modulation Order in Rayleigh Channel for the Far User

BER performance of the proposed scheme for the far user is given in Fig. 8. In this figure, the curve colored with green represents the error that occurs during the transmission of ANM bits sub-stream, while the curve colored with pink is the error that occurs during the transmission of main bits substream, which is modulated by the conventional PSK/QAM modulation. By calculating the average values of these two curves the average BER result for the far user can be obtained as shown in the cyan colored curve.

It should be stated that the characteristics of the far user's BER show similarities with the conventional MIMO-ANMAAS. The reason behind this is that the antennas, which are deployed for the near user's data are activated in a low power compared with the antennas that are deployed for the far user's data. This leads to the near user's data to be faded at the far user's location, which results in the far user not to be affected by the near user's data, and dependently not to be affected by any additional interference. Thus, the system works in the same way as the conventional MIMO-ANMAAS for the far user.

Fig. 9 illustrates the BER performances of the far user and near user for the proposed scheme. The blue colored curve represents the BER performance of the far user, which is obtained by calculating the average value of the BER

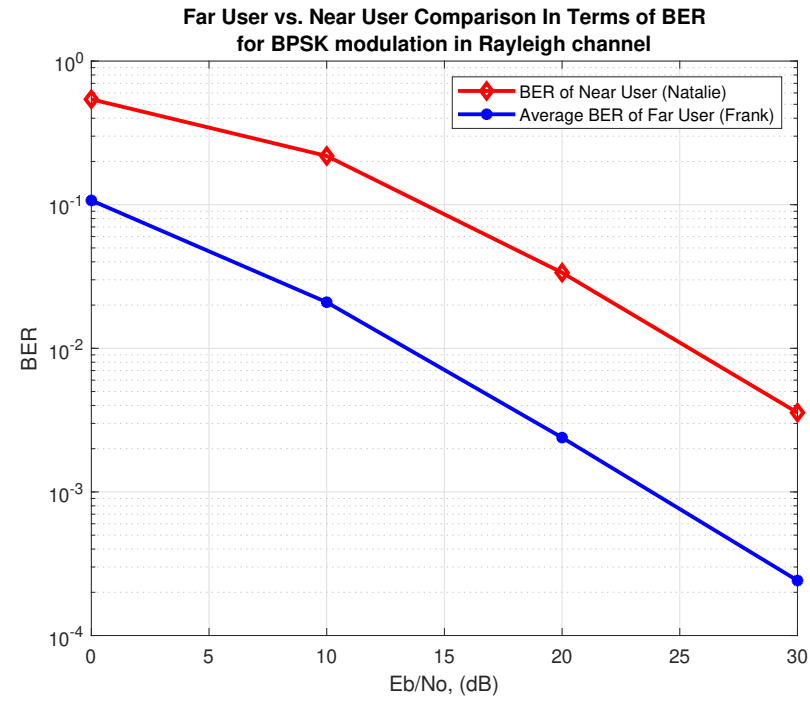

FIGURE 9. Simulated BER Comparison Between Far User and Near User for the Proposed MU-MIMO-ANM-AAS Scheme Under BPSK Modulation Order in Rayleigh Channel

results of main bits and ANM bits sub-streams. On the other hand, the red colored curve represents the performance of near user in terms of BER. As it can be seen from the Fig. 9, BER result of the near user exhibits lower performance in comparison with the far user. This is caused by the fact that the transmission priority is given to the far user by implementing the ANM technique with the highest possible channel capacity offering antennas in high power, while the near user is served by the inactive antennas for the far user with low channel capacity in low power. However, as it is seen by the Fig. 9, obtained performance of the near user's BER is getting better as the SNR value increases and it can manage to pass $10^{-2}$ when the SNR is 30 .

Lastly, Fig. 10 is the exhibition of the derived BER formulas for MU-MIMO-ANM-AAS, where the overall average theoretical BER performance indicated in blue color curve for the far user, and the theoretical BER performance indicated in black color curve for the near user show similar characteristics with the simulated BER performances that is shown in Fig. 9.

\section{CONCLUSION}

In this paper, a novel data transmission scheme MU-MIMOANM-AAS is introduced to implement the merits of ANM into multi-user cases. The adopted idea to achieve this purpose is to utilize the number of antennas to transmit additional data bits along with the conventional $M$-ary PSK/QAM symbols with the highest channel capacity offering antennas for the transmission of far user's data and deploy the remaining low channel capacity offering antennas to serve another user who is located in a nearer location to transmitter and expects to be served by a different data set. The advantage of the proposed technique is that, since the 


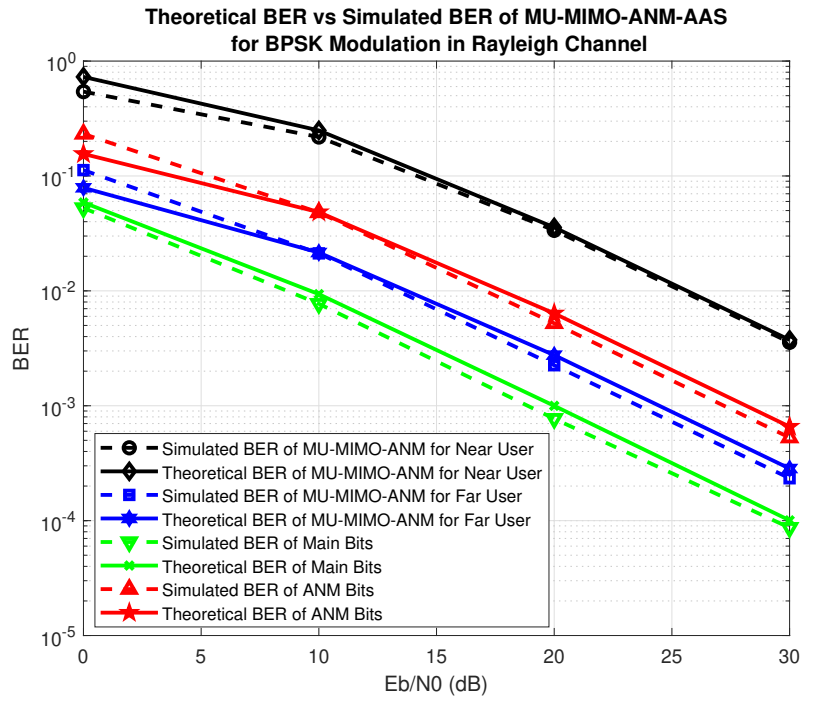

FIGURE 10. Theoretical BER Results of the Proposed Scheme MU-MIMO-ANM-AAS Under BPSK Modulation Order in Rayleigh Channel

ANM does not dictate the indices of the antennas but rather dictate the dictates the number of antennas, the far user can be served in the best possible way in terms of BER performance and spectral efficiency by exploiting the adaptive antenna selection capability of ANM, while the unused sources of the transmitter are not wasted and utilized to serve a near user instead of remaining inactive, as the far user does not use all the transmit antennas for transmission at a time.

\section{REFERENCES}

[1] J. Hoydis, S. Ten Brink, and M. Debbah, "Massive MIMO in the ul/dl of cellular networks: How many antennas do we need?" IEEE Journal on selected Areas in Communications, vol. 31, no. 2, pp. 160-171, 2013.

[2] E. Björnson, L. Sanguinetti, J. Hoydis, and M. Debbah, "Designing multiuser MIMO for energy efficiency: When is massive MIMO the answer?" in 2014 IEEE Wireless Communications and Networking Conference (WCNC). IEEE, 2014, pp. 242-247.

[3] N. Su, E. Panayirci, M. Koca, A. Yesilkaya, H. V. Poor, and H. Haas, "Physical layer security for multi-user MIMO visible light communication systems with generalized space shift keying," IEEE Transactions on Communications, 2021.

[4] M. Kirik and J. M. Hamamreh, "Multiple MIMO with joint block antenna number modulation and adaptive antenna selection for future wireless systems," RS Open Journal on Innovative Communication Technologies, vol. 1, no. 2, 122020.

[5] — , "Multiple MIMO with antenna number modulation," in 2020 International Conference on UK-China Emerging Technologies (UCET). IEEE, 2020, pp. 1-4.

[6] J. Mietzner, R. Schober, L. Lampe, W. H. Gerstacker, and P. A. Hoeher, "Multiple-antenna techniques for wireless communications-a comprehensive literature survey," IEEE communications surveys \& tutorials, vol. 11, no. 2, pp. 87-105, 2009.

[7] H. Huang, C. B. Papadias, and S. Venkatesan, MIMO communication for cellular networks. Springer Science \& Business Media, 2011.

[8] D. Wang, J. Wang, X. You, Y. Wang, M. Chen, and X. Hou, "Spectral efficiency of distributed MIMO systems," IEEE Journal on Selected Areas in Communications, vol. 31, no. 10, pp. 2112-2127, 2013.

[9] S. Narayanan, M. J. Chaudhry, A. Stavridis, M. Di Renzo, F. Graziosi, and H. Haas, "Multi-user spatial modulation MIMO," in 2014 IEEE Wireless Communications and Networking Conference (WCNC). IEEE, 2014, pp. 671-676.
[10] D. Gesbert, M. Kountouris, R. W. Heath, C.-B. Chae, and T. Salzer, "Shifting the MIMO paradigm," IEEE signal processing magazine, vol. 24, no. 5, pp. 36-46, 2007.

[11] S. Serbetli and A. Yener, "Transceiver optimization for multiuser MIMO systems," IEEE Transactions on Signal Processing, vol. 52, no. 1, pp. 214 226, 2004.

[12] H. Q. Ngo, E. G. Larsson, and T. L. Marzetta, "Energy and spectral efficiency of very large multiuser MIMO systems," IEEE Transactions on Communications, vol. 61, no. 4, pp. 1436-1449, 2013.

[13] G. Caire, N. Jindal, M. Kobayashi, and N. Ravindran, "Multiuser MIMO achievable rates with downlink training and channel state feedback," IEEE Transactions on Information Theory, vol. 56, no. 6, pp. 2845-2866, 2010.

[14] J. Jose, A. Ashikhmin, T. L. Marzetta, and S. Vishwanath, "Pilot contamination and precoding in multi-cell tdd systems," IEEE Transactions on Wireless Communications, vol. 10, no. 8, pp. 2640-2651, 2011.

[15] G. Durisi and S. Benedetto, "Performance evaluation of th-ppm uwb systems in the presence of multiuser interference," IEEE Communications Letters, vol. 7, no. 5, pp. 224-226, 2003.

[16] H. Suzuki, "Multiuser interference canceler for ds-cdma system," Jun. 24 2003, uS Patent 6,584,115.

[17] M. Costa, "Writing on dirty paper (corresp.)," IEEE transactions on information theory, vol. 29, no. 3, pp. 439-441, 1983.

[18] Z. Shen, R. Chen, J. G. Andrews, R. W. Heath, and B. L. Evans, "Low complexity user selection algorithms for multiuser MIMO systems with block diagonalization," IEEE Transactions on Signal Processing, vol. 54 no. 9, pp. 3658-3663, 2006.

[19] L. Liang, W. Xu, and X. Dong, "Low-complexity hybrid precoding in massive multiuser MIMO systems," IEEE Wireless Communications Letters, vol. 3, no. 6, pp. 653-656, 2014.

[20] P. Li, R. C. De Lamare, and R. Fa, "Multiple feedback successive interference cancellation detection for multiuser MIMO systems," IEEE Transactions on Wireless Communications, vol. 10, no. 8, pp. 2434-2439, 2011.

[21] L. Ping, "Interleave-division multiple access and chip-by-chip iterative multi-user detection," IEEE Communications Magazine, vol. 43, no. 6, pp S19-S23, 2005.

[22] H. V. Poor, "Iterative multiuser detection," IEEE Signal Processing Magazine, vol. 21, no. 1, pp. 81-88, 2004.

[23] J. Lee and N. Jindal, "High snr analysis for MIMO broadcast channels: Dirty paper coding versus linear precoding," IEEE Transactions on Information Theory, vol. 53, no. 12, pp. 4787-4792, 2007.

[24] Z. Tu and R. S. Blum, "Multiuser diversity for a dirty paper approach," IEEE Communications letters, vol. 7, no. 8, pp. 370-372, 2003.

[25] H. Zhang and H. Dai, "Cochannel interference mitigation and cooperative processing in downlink multicell multiuser MIMO networks," EURASIP Journal on Wireless Communications and Networking, vol. 2004, no. 2, pp. 1-14, 2004.

[26] L. Zhao, D. W. K. Ng, and J. Yuan, "Multi-user precoding and channel estimation for hybrid millimeter wave systems," IEEE Journal on Selected Areas in Communications, vol. 35, no. 7, pp. 1576-1590, 2017.

[27] C. Windpassinger, R. F. Fischer, T. Vencel, and J. B. Huber, "Precoding in multiantenna and multiuser communications," IEEE Transactions on Wireless Communications, vol. 3, no. 4, pp. 1305-1316, 2004.

[28] J. M. Hamamreh, M. KIRIK, M. O. SAGMAN, and N. ISHIKAWA "Multiple input multiple output with antenna number modulation and adaptive antenna selection," RS Open Journal on Innovative Communication Technologies, vol. 1, no. 1, 2020.

[29] R. Y. Mesleh, H. Haas, S. Sinanovic, C. W. Ahn, and S. Yun, "Spatial modulation," IEEE Transactions on vehicular technology, vol. 57, no. 4, pp. 2228-2241, 2008.

[30] J. Jeganathan, A. Ghrayeb, and L. Szczecinski, "Spatial modulation: Optimal detection and performance analysis," IEEE Communications Letters, vol. 12 , no. 8, pp. 545-547, 2008.

[31] M. Di Renzo, H. Haas, and P. M. Grant, "Spatial modulation for multipleantenna wireless systems: A survey," IEEE Communications Magazine, vol. 49, no. 12, pp. 182-191, 2011. 
SEYIT KARATEPE received the B.Sc. degree in electrical and electronics engineering from Antalya Bilim University, Turkey in 2020. He is currently pursuing his M.Sc degree in Antalya Bilim University in the electrical and computer engineering department. His current research interests include orthogonal frequency division multiplexing multiple input multiple output systems, multi-dimensional modulation techniques, and orthogonal/non-orthogonal multiple access schemes for future wireless systems.

MUHAMMET KIRIK received the B.Sc. degree in electrical and electronics engineering from Antalya Bilim University, Turkey in 2020. He is currently pursuing his M.Sc degree in Antalya Bilim University in the electrical and computer engineering department. His current research interests include orthogonal frequency division multiplexing multiple input multiple output systems, multi-dimensional modulation techniques, and orthogonal/non-orthogonal multiple access schemes for future wireless systems.

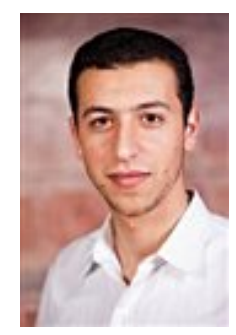

JEHAD M. HAMAMREH received the B.Sc. degree in electrical and telecommunication engineering from An-Najah University, Nablus, in 2013, and the Ph.D. degree in electrical electronics engineering and cyber systems from Istanbul Medipol University, Turkey, in 2018. He was a Researcher with the Department of Electrical and Computer Engineering, Texas AM University. $\mathrm{He}$ is currently an Assistant Professor with the Electrical and Electronics Engineering Department, Antalya International (Bilim) University, Turkey. His current research interests include wireless physical and MAC layers security, orthogonal frequency-division multiplexing multiple-input multiple-output systems, advanced waveforms design, multi-dimensional modulation techniques, and orthogonal/non-orthogonal multiple access schemes for future wireless systems. He is a Regular Reviewer for various peer-reviewed journals as well as a TPC Member for several international conferences. He is very open for collaboration and can be reached via email at jehad.hamamreh@gmail.com. 